\title{
Ultralong Spin Lifetime in Light Alkali Atom Doped Graphene
}

\author{
B. G. Márkus, P. Szirmai, K. F. Edelthalhammer, P. Eckerlein, A. Hirsch, F. Hauke, N. M. Nemes, \\ Julio C. Chacón-Torres, B. Náfrádi, L. Forró, T. Pichler, and F. Simon*
}

Cite This: ACS Nano 2020, 14, 7492-7501

Read Online

ACCESS | L W Metrics \& More | 国 Article Recommendations | st Supporting Information

ABSTRACT: Today's great challenges of energy and informational technologies are addressed with a singular compound, Liand $\mathrm{Na}$-doped few-layer graphene. All that is impossible for graphite (homogeneous and high-level $\mathrm{Na}$ doping) and unstable for single-layer graphene works very well for this structure. The transformation of the Raman $G$ line to a Fano line shape and the emergence of strong, metallic-like electron spin resonance (ESR) modes attest the high level of graphene doping in liquid ammonia for both kinds of alkali atoms. The spin-relaxation time in our materials, deduced from the ESR line width, is 6-8 ns, which is comparable to the longest values found in spin-
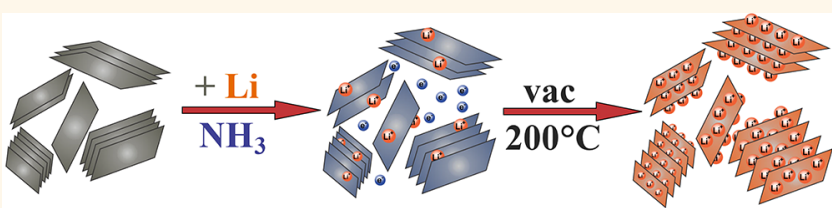
transport experiments on ultrahigh-mobility graphene flakes.

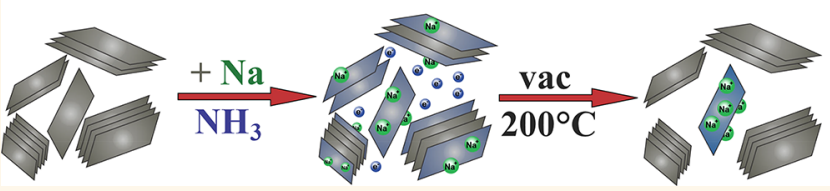
This could qualify our material as a promising candidate in spintronics devices. On the other hand, the successful sodium doping, this being a highly abundant metal, could be an encouraging alternative to lithium batteries.

KEYWORDS: few-layer graphene, alkali atom doping, Raman spectroscopy, electron spin lifetime, electron spin resonance

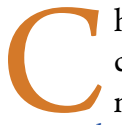

harge state in various forms of carbon can be conveniently controlled using alkali atom doping methods. It led to applications in, for example, energy storage $^{1}$ and to the discovery of compelling correlated phases such as superconductivity (with $T_{\mathrm{c}}=11.5 \mathrm{~K}$ in $\mathrm{CaC}_{6}{ }^{2,3}$ and $T_{\mathrm{c}}$ $=28 \mathrm{~K}$ in $\mathrm{Rb}_{3} \mathrm{C}_{60}{ }^{4}$ ), spin density waves in fullerides, ${ }^{5}$ and the Tomonaga-Luttinger to Fermi liquid crossover in single-wall carbon nanotubes. 6

Control over the number of free electrons in carbon could be also exploited in spintronics, ${ }^{8-10}$ i.e., when using the electron spin as an information carrier and storage unit. Doping-induced metallic carbon has the smallest spin-orbit coupling besides metallic $\mathrm{Li}$ and $\mathrm{Be}$, which is expected to result in ultralong spin lifetime, $\tau_{\mathrm{s}}$, a prerequisite for spintronics. Graphene arose as a promising candidate for spintronics purposes due to the predicted long $\tau_{s}$; its actual value is controversial according to spin transport studies, ${ }^{11-16}$ and it ranges from $100 \mathrm{ps}^{11}$ up to $12 \mathrm{~ns}^{12,15,16}$ with theoretical hints that the short lifetime originates from extrinsic effects. ${ }^{17}$ Doping graphene with light alkali atoms would enable the accurate determination of $\tau_{\mathrm{s}}$ for the itinerant electrons by spin spectroscopy, i.e., electron spin resonance (ESR). ${ }^{18,19}$ Light alkali atoms have a small spin-orbit coupling; ${ }^{20,21}$ thus the intrinsic spin lifetime in graphene is expected to be observed with this approach.
Conventional alkali doping of carbon proceeds in the socalled vapor phase, which works well for heavier alkali atoms with a lower melting point $(\mathrm{K}, \mathrm{Rb}$, and $\mathrm{Cs}):^{22}$ the alkali atoms are heated together with the desired form of carbon (graphite, graphene, nanotubes, or fullerene). For $\mathrm{Li}$ and $\mathrm{Ca}$ doping, graphite doping was achieved by immersing the sample into molten $\mathrm{Li}$ or $\mathrm{Li} / \mathrm{Ca}$ mixtures ${ }^{2}$ with temperatures up to $350{ }^{\circ} \mathrm{C}$. This relatively high temperature is dictated by the melting point and is due to the slow kinetics of the diffusion process, but the reaction has a small temperature window due to formation of alkali carbides around $450{ }^{\circ} \mathrm{C}$. This method can only be formed for a bulky sample (e.g., for a piece of HOPG or graphite single crystal) that can be inserted and removed from the molten metal.

Alkali atom doping of graphene is also intensively studied. $^{23-29}$ Achieving controllable and high-level doping would be particularly important for chemically exfoliated graphene, which is the bulk form of graphene, e.g., for energy

Received: April 16, 2020

Accepted: June 2, 2020

Published: June 2, 2020

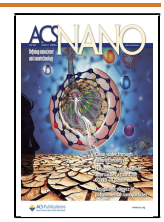


a)
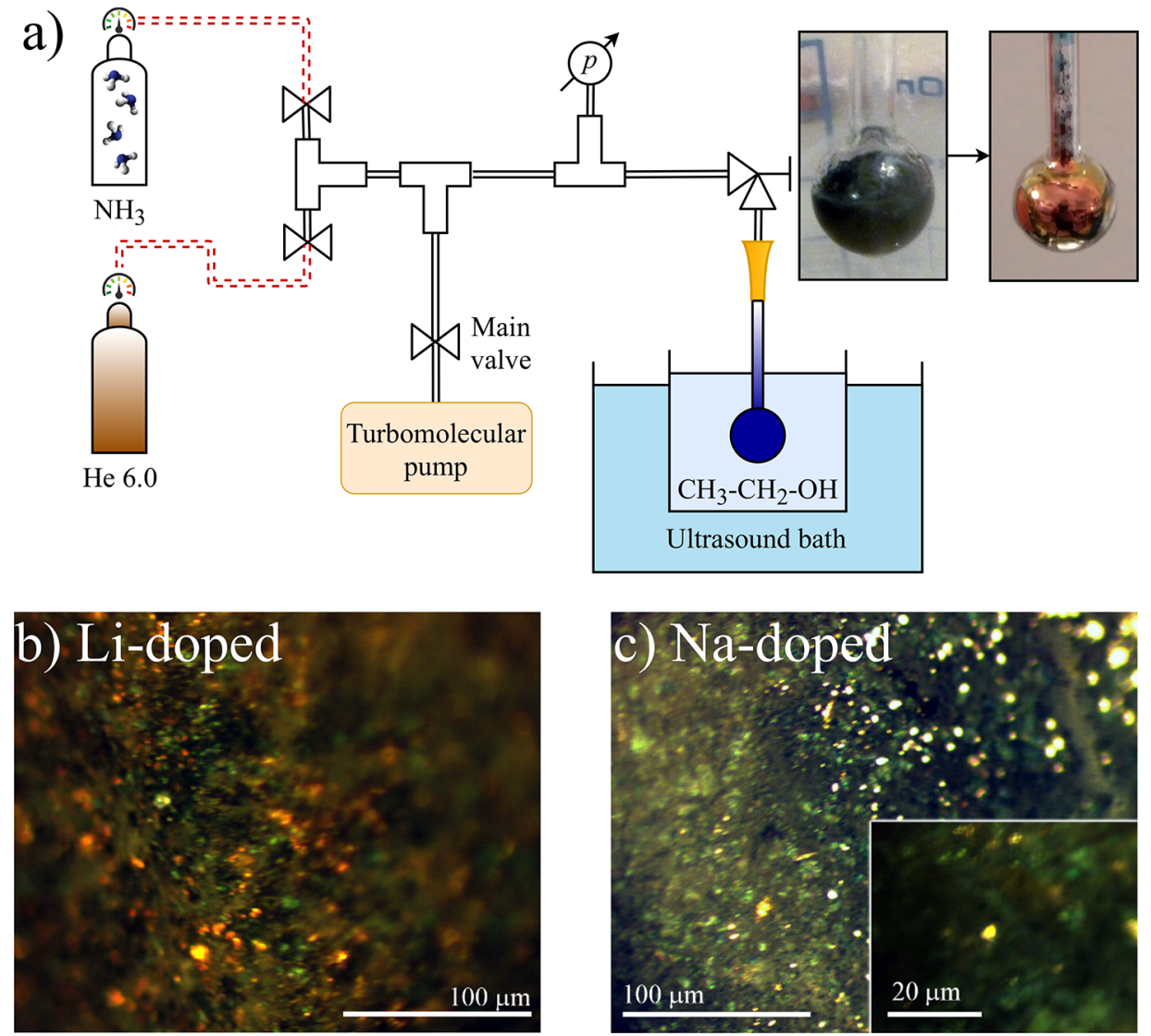

Figure 1. (a) Schematics of the doping setup connected to a vacuum line with gas inlets. The alkali-ammonia solution (blue bubble) is kept around $-40{ }^{\circ} \mathrm{C}$ using an ethanol bath. A bath sonicator assists the doping, and it surrounds the ethanol container. Inset shows the photograph of the solution at the beginning and at the end of the process. (b and c) Microscope images of the final materials. Note that Li doping results in bright, yellow-brownish and bluish flakes, while upon $\mathrm{Na}$ doping only bluish areas are present. Inset in (c) shows a smaller area of the sodium-doped sample shot with a $50 \times$ objective to emphasize the presence of bluish areas.

storage purposes. ${ }^{30-32}$ Especially Na-doped graphene is an important candidate to replace conventional $\mathrm{Li}$-ion batteries due to the low cost and high abundance of sodium. ${ }^{33-36}$

However, $\mathrm{Na}$ is somewhat of an outlier among the alkali metals, as it does not intercalate graphite under ambient pressure except for a very low stoichiometry of $\mathrm{NaC}_{64}{ }^{37-41}$ as compared to, for example, $\mathrm{LiC}_{6}$ and $\mathrm{KC}_{8}$. Successful $\mathrm{Na}$ doping was reported for graphene, prepared by a solvothermal synthesis by the Choucair method, ${ }^{42}$ either using electrochemical doping ${ }^{43}$ or when the material was synthesized from Na-containing precursors. ${ }^{44}$ However, solvothermal-derived graphene is known to have a relatively large defect concentration as well as a limited flake size; it is thus intriguing whether $\mathrm{Na}$ doping could be achieved for a highquality and high-area mono- or few-layer graphene.

As mentioned, doping with light alkali atoms cannot be performed with the vapor phase method. An alternative doping route uses solvents, such as liquid ammonia and organic solvents (e.g., tetrahydrofuran (THF) ${ }^{45}$ or 2-methyltetrahydrofuran (2-MeTHF) and 2,5-dimethyltetrahydrofuran (diMeTHF $)^{46,47}$ ). These are known to dissolve well the alkali and some alkaline-earth elements. Then, the reaction between the alkali atoms and the carbon material proceeds in the solution at moderate temperatures and with a high efficiency due to the large reaction surface. This procedure was used to obtain highly doped fullerides ${ }^{4-53}$ and carbon nanotubes (nanotubides). ${ }^{54}$ This route is promising for doping graphene with light alkali atoms, and it could address the value of spin lifetime of itinerant electrons and whether $\mathrm{Na}$ can differentiate between mono- and few-layer graphene, as the latter is known to be inevitably present in chemically exfoliated graphene. ${ }^{55}$

Here, we report the synthesis of $\mathrm{Li}$ - and $\mathrm{Na}$-doped few-layer graphene (FLG) using liquid ammonia. The FLG material was prepared by chemical exfoliation. Raman spectroscopy, conductivity, and electron spin resonance studies indicate a successful doping. The strongly Fano-like Raman line shapes evidence a sizable electron-phonon coupling. However, neither SQUID magnetometry nor conductivity measurements gave evidence for a superconducting phase down to $2 \mathrm{~K}$. The spin-relaxation lifetime of conduction electrons is in excess of 7 ns, which represents an ultralong value especially for chemically exfoliated graphene. We argue that $\mathrm{Na}$ dopes selectively monolayer graphene, as a few-layer graphene resembles graphite, for which $\mathrm{Na}$ doping is known to be impossible.

\section{RESULTS AND DISCUSSION}

Few-layer graphene samples were doped with lithium and sodium in liquid ammonia with the setup shown in Figure 1a. We previously demonstrated that the starting material mainly consists of graphene flakes with 5 or less layers with a lognormal distribution centered around 3 layers. ${ }^{55}$ Photographs of the samples are presented in Figure $1 \mathrm{~b}$ and c. A characteristic color change was observed to the black FLG material upon 
doping, which is a fingerprint of charge transfer from the alkali atoms toward the carbon, as seen before for graphite intercalation compounds $^{22,56}$ and graphene. ${ }^{23,24,55}$ In the case of lithium, both yellow-brownish and bluish spots are present, which attests to a successful doping of the material. For sodium, only bluish areas are observable, which hints at lighter, yet successful doping. For intercalated graphite, a yellow color is the signature of the highest level of doping (also referred to as stage-I compounds), whereas a blue color is a signature of a lower level of doping. 22

The most striking observation of this synthesis process is the presence of any color change to the Na-doped FLG sample. $\mathrm{Na}$ is known to intercalate (or dope) graphite to a very low level such as $\mathrm{NaC}_{64}{ }^{37-40}$ which has the same color as graphite. In fact, a higher level of $\mathrm{Na}$ doping can only be achieved with an ultrahigh-pressure synthesis method, ${ }^{57}$ but the resulting material is unstable at ambient pressure. It has long been intriguing why $\mathrm{Na}$ does not effectively dope graphite. Graphite intercalation compounds (GICs) possess a long-range ordered structure for the alkali atoms and also for the graphene layers that are adjacent to layers of alkali atoms. Recent $a b$ initio calculations ${ }^{41}$ hint at a delicate interplay between the alkali ion induced structural deformation (and the corresponding weakening in the van der Waals interaction between graphene layers) and the covalent (for $\mathrm{Li}$ ) versus ionic (for $\mathrm{Na}$ ) interaction, which makes the formation of Na-intercalated graphite thermodynamically unfavored. In contrast, sodium can effectively dope fullerides up to $\mathrm{Na}_{4} \mathrm{C}_{60},{ }^{58-60}$ where the voids between the fullerene balls can be conveniently filled; thus the deformation energy of the van der Waals molecular crystal is smaller and is compensated by the energy gain during the electron charge transfer. Therefore, it is expected that $\mathrm{Na}$ could dope monolayer graphene where the energy loss due to deformation is also absent.

Given the surprising result of a successful and relatively high level of $\mathrm{Na}$ doping of the few-layer graphene sample, we performed Raman spectroscopy on the synthesized materials, as it is a sensitive probe of the charge transfer toward the carbonaceous material. Raman spectra recorded at $514.5 \mathrm{~nm}$ wavelength of the materials are presented in Figure 2. Raman spectrum of the pristine material displays the usual D $\left(1358 \mathrm{~cm}^{-1}\right), \mathrm{G}\left(1580 \mathrm{~cm}^{-1}\right)$, and $2 \mathrm{D}\left(2710 \mathrm{~cm}^{-1}\right)$ bands, and it reproduces the earlier reports on similar samples. ${ }^{61}$

The lithium-doped FLG sample shows a broad and intensive Fano-shaped line (also referred to as Breit-Wigner-Fano, or BWF, line shape $)^{62,63}$ at $1507 \mathrm{~cm}^{-1}$, which dominates the spectrum. The $\mathrm{D}$ and $2 \mathrm{D}$ modes are barely visible, and the latter is extremely broadened. The Fano line is downshifted and flattened compared to the original G mode, as expected upon heavy doping. Compared to the Raman spectrum of $\mathrm{LiC}_{6}$ GIC, the observed peak is relatively close to its $E_{2 g_{1}}$ mode at $1546 \mathrm{~cm}^{-1}$. The doped samples are in a quartz tube, which explains the origin of the Raman line denoted by an asterisk.

We also present Raman data for two Na-doped samples, one denoted as heavily, the other as moderately doped. The heavy doping notation refers to the highest achievable doping level, whereas the moderate doping is representative for a sample prepared with a smaller amount of alkali dopants. Both types of sodium-doped samples present a Fano line at around $1572 \mathrm{~cm}^{-1}$ and a symmetric Lorentzian peak at 1600$1602 \mathrm{~cm}^{-1}$. The Fano peak at the lower Raman shift confirms the presence of highly intercalated flakes in agreement with the

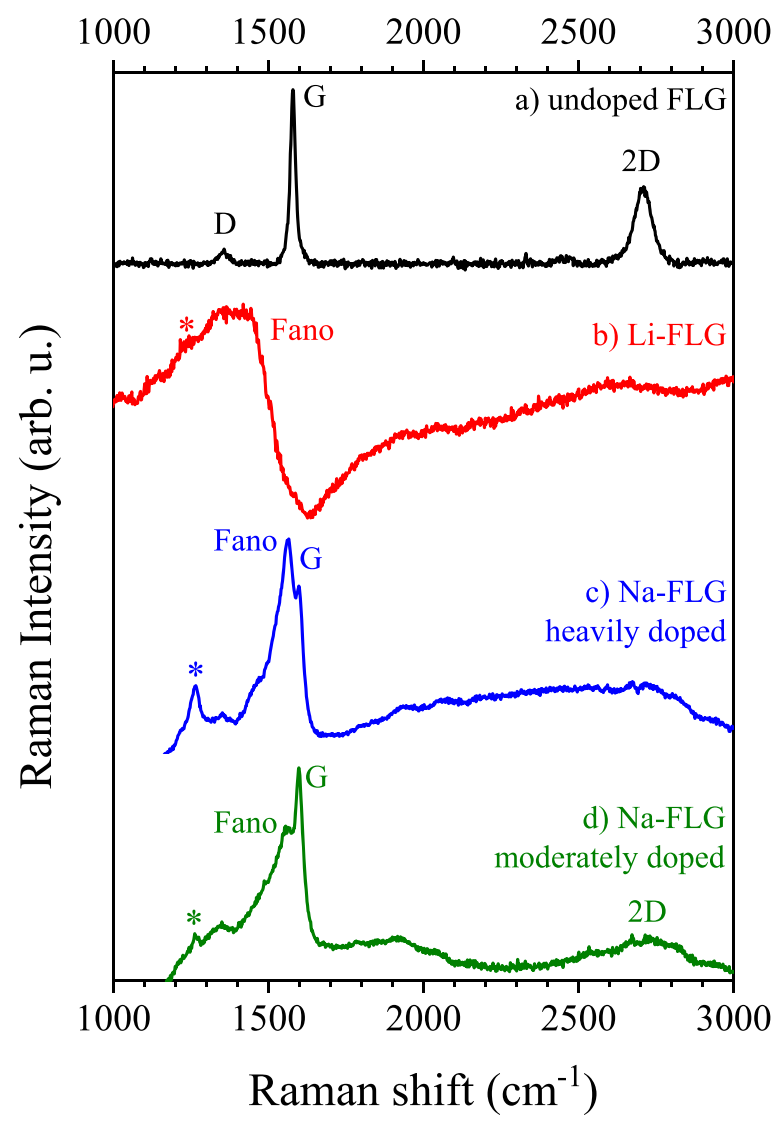

Figure 2. Raman spectra taken at $514.5 \mathrm{~nm}$ wavelength. (a) Undoped FLG shows the usual D, G, and 2D modes. (b) A broad and intensive Fano line at $1510 \mathrm{~cm}^{-1}$ dominates the spectrum in the lithium-doped FLG sample; the D and 2D modes are barely visible, and the latter is extremely broadened. (c, d) Sodium-doped samples also display a Fano line at $1566 \mathrm{~cm}^{-1}$ and a symmetric peak at $1600 \mathrm{~cm}^{-1}$. The latter is denoted by $G$ and is associated with weakly charged graphene flakes. Asterisk denotes a peak from the quartz sample holder.

above-mentioned surprising microscopic observation, i.e., that significant charge transfer is observed in the $\mathrm{Na}$-doped samples. The Fano line shape in alkali-metal intercalation compounds with graphite only occurs when a stage-I compound is reached; that means when each graphene layer is surrounded from both sides by an alkali atom layer. ${ }^{64}$ The observation of this asymmetric mode in the Na-doped sample provides a direct proof for the existence of $\mathrm{Na}$-doped monolayer graphene. The symmetric peak at higher Raman shifts (denoted by G) is associated with weakly charged or incompletely intercalated flakes. ${ }^{40}$ The $2 \mathrm{D}$ mode is very broad in all samples, as expected for a high level of doping. ${ }^{56,64}$

In general, the Fano line shape is the result of quantum interference between the zone-center phonons and the electronic transitions; ${ }^{62,63}$ thus it is an important benchmark of a significant charge transfer in carbonaceous materials including fullerides, ${ }^{63}$ carbon nanotubes (nanotubides), ${ }^{66}$ graphite, ${ }^{67}$ and graphene (graphenides). ${ }^{23,24,27}$

Additional information about the electronic structure, the level of charge transfer, and the magnitude and sign of the electron-phonon coupling can be obtained from the details of the Fano line shape. According to refs 62 and 63 the line shape reads as a function of the Raman shift energy, $\hbar \omega$ : 


$$
I(\omega)=I_{0} \frac{(q+\bar{\epsilon})^{2}}{1+\bar{\epsilon}^{2}}+A
$$

where $q$ is the Fano asymmetry line shape parameter, $\epsilon=\frac{\omega-\omega_{0}-\Delta}{\Gamma / 2}$, and $A$ is an offset parameter. Herein $\hbar \omega_{0}$ is the vibrational energy of the unperturbed phonon, $\hbar \Delta$ is the interaction-induced shift of the phonon energy, and $\hbar \Gamma$ is the broadening parameter due to the interaction. In general $\Gamma$ and $\Delta$ increase with the strength of the electron-phonon coupling; however $q$ is inversely proportional to it, $q= \pm \infty$ represents the noninteracting limit, and $q \rightarrow 0$ is the limit of strong electron-phonon interaction.

We found $q=-1.03$ for the Li-doped FLG and $q=-2.64$ and $q=-3.17$ for the heavily and moderately Na-doped FLG samples, respectively (details of the line shape analysis are provided in the Supporting Information). These figures have to be compared with $q=-1.09$ found for the graphite intercalation compounds ${ }^{56} \mathrm{LiC}_{6}, \mathrm{KC}_{8}$, and $\mathrm{CaC}_{6}$, of which the latter is a superconductor with $T_{\mathrm{c}}=11.5 \mathrm{~K}^{68}$

The significant charge transfer and the sizable electronphonon coupling in the Li-doped FLG motivated us to search for traces of superconductivity for both the $\mathrm{Li}$ - and $\mathrm{Na}$-doped materials. However, SQUID magnetometry in a zero field cooled condition and using a 10 Oe of sensing field, while warming the samples from $2 \mathrm{~K}$, did not reveal any traces of superconductivity.

Electron spin resonance spectroscopy ${ }^{69}$ can provide a great deal of information about the electrons in a material, including localized electron spins, which are related to defects or dangling bonds, and also about delocalized, i.e. conduction, electron species. Figure 3 presents the ESR spectra of the undoped and Li- and Na-doped FLG samples at 230 and $50 \mathrm{~K}$. In each cases, the observed line shape can be decomposed into two separate lines. In the undoped material, the lines are completely symmetric and thus can be fitted with derivative Lorentzians. The origin of these lines is most probably dangling bonds or lattice defects, which give rise to a paramagnetic signal. The presence of such lines is very common in carbon materials including fullerenes, ${ }^{70}$ carbon nanotubes and graphite, ${ }^{71}$ graphene, ${ }^{, 2}$ and boron-doped diamond. $^{73}$

Upon doping the FLG sample with $\mathrm{Li}$ and $\mathrm{Na}$, the ESR spectra change significantly: strong signals with about 30-50 times larger intensity appear with an asymmetric line shape. An asymmetric ESR line shape is identified as a so-called Dysonian line, ${ }^{19}$ which is the usual case in metallic materials. In fact, the Dysonian line shape in ESR can be considered as analogous to the Fano line shape in Raman spectroscopy; however its physical origin is different. The Dysonian line shape is due to the varying microwave phase along the sample volume due to the limited penetration of microwaves. The asymmetry is stronger for both lines in the lithium-doped material than in the sodium-doped one. This indicates a smaller microwave penetration depth and thus a larger conductivity. This observation is in agreement with the visual observations and the Raman spectroscopy results above, i.e., that charge transfer is stronger in the lithium-doped sample.

Figure 3 shows that the ESR signal in the alkali-doped samples can be decomposed into two distinct ESR lines with different line widths. The presence of multiple ESR lines is often encountered in alkali atom doped carbon materials. The examples include alkali-doped fullerides ${ }^{70,74}$ and carbon

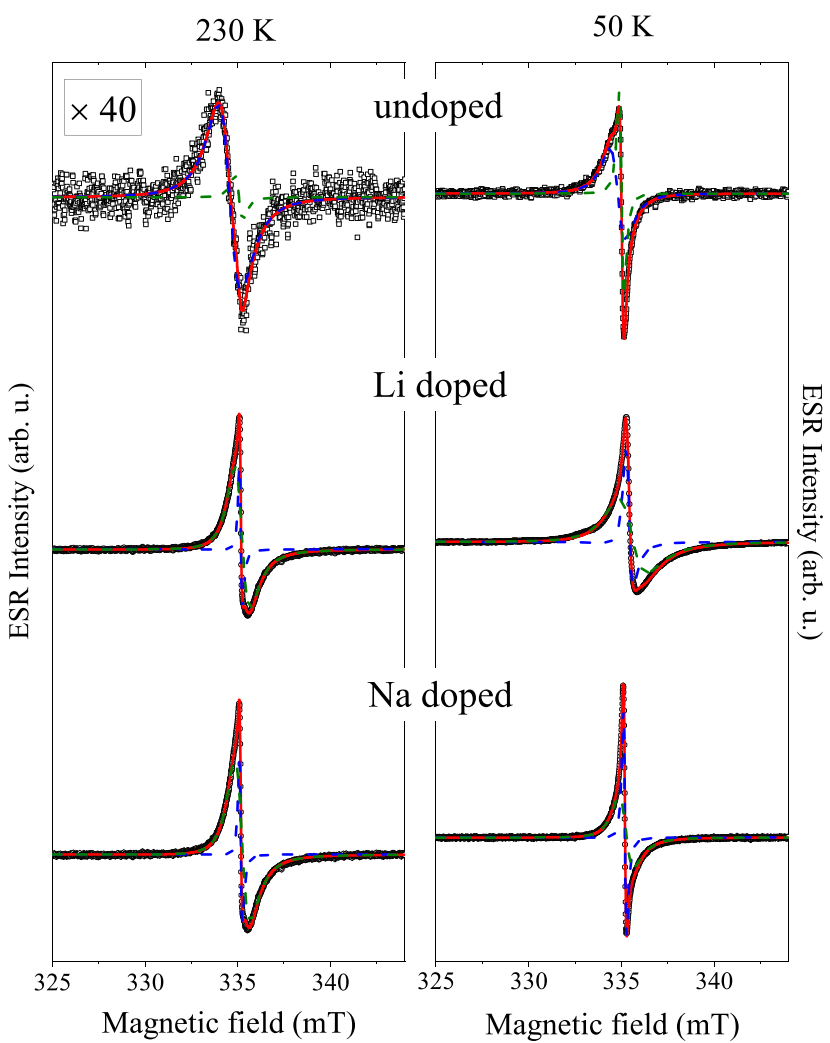

Figure 3. ESR spectra of the undoped and Li- and Na-doped FLG samples at 230 and $50 \mathrm{~K}$. Open symbols are the measured data, and the fitted curve is represented with a continuous red line. Blue and green curves are the decomposition to the two components present for each sample. The two lines are fully symmetric in the undoped material, whereas both components are asymmetric in the doped ones, indicating a metallic sample. Note the zoomed vertical scale for the undoped material.

nanotubes (nanotubides)..$^{54,75}$ The origin of multiple ESR lines in doped carbon could be an inhomogeneous doping or the presence of localized paramagnetic spins.

To gain a deeper insight into the electronic properties of the doped materials, the temperature dependence of the ESR signal was studied in the 5 to $250 \mathrm{~K}$ temperature range. The ESR intensity is directly proportional to the spin susceptibility, which allows identifying the nature of spins (localized or delocalized) which give rise to the ESR signal. In addition, the ESR line width is related to $T_{2}$, which is called the spin-spin relaxation time (due to historical reasons). ${ }^{18,19,69}$ In the following, we refer to this as spin-relaxation time, or $\tau_{\mathrm{s}}$, to conform with the spintronics literature. Determining $\tau_{\mathrm{s}}$ is directly relevant for the spintronics applications of graphene.

The spectroscopic properties, including line intensity, position, and line width, were determined by fitting the Dysonian lines with a mixture of absorption and dispersion Lorentzian lines, ${ }^{76,77}$ which is a valid approach when the conduction electrons are diffusing through the microwave penetration depth slowly (this is the so-called NMR limit) compared to their lifetime. ${ }^{19}$

The temperature-dependent ESR intensity normalized by the sample mass and the ESR line width is shown in Figure 4. It reinforces the earlier observation that the observed spin susceptibility increases significantly upon doping. In addition, the temperature-dependent character of the spin susceptibility also characteristically changes: in the undoped sample, the spin 


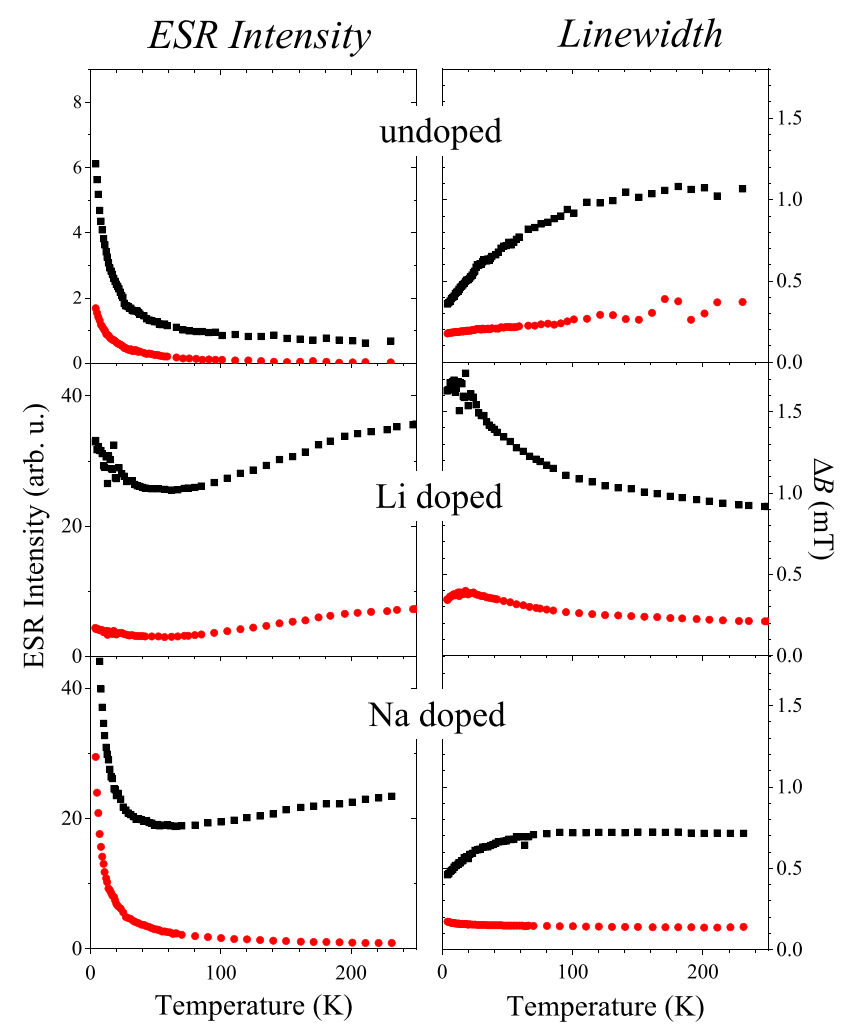

Figure 4. Temperature-dependent ESR intensity (normalized by the sample mass) and line width. Note the significantly smaller scale for the undoped material. The lithium-doped sample shows two Pauli-like signal intensities, which is a clear indication of a metallic behavior. On the other hand, the sodium-doped material shows a mixed behavior with one Curie and one Pauli-like signal.

susceptibility shows a significant increase upon lowering the temperature, which is characteristic for localized, i.e., paramagnetic, spin species. We thus term it a "Curie-like" behavior, where $\chi_{\mathrm{s}} \approx 1 / T$. In fact, the intensity of the broader peak in the undoped material remains finite at higher temperature. This can be related to an unintentional doping due to some residual solvent or contamination in the starting material.

In contrast, a Pauli-like spin susceptibility, i.e., with little temperature dependence, dominates the signal for both the lithium- and sodium-doped samples above $20 \mathrm{~K}$, although the sodium-doped material also contains a Curie-like contribution with a much smaller intensity. An ESR signal with a spin susceptibility with little or no temperature dependence is characteristic for conduction electrons. The fact that the $\mathrm{Li}$ doped material contains two such signals hints that the doping is inhomogeneous. We note that the presence of unreacted metallic particles can be excluded as the origin of the observed metallic signals, since metallic $\mathrm{Li}$ and $\mathrm{Na}$ have characteristic line shapes (typical for strongly diffusing electrons), which is not observed herein. ${ }^{18,19}$ Even the Pauli-like signal shows an upturn in intensity in the Na-doped sample below $20 \mathrm{~K}$; similar effects are often encountered when a minute amount (of the $10 \mathrm{ppm} \mathrm{level/lattice} \mathrm{sites)} \mathrm{of} \mathrm{paramagnetic} \mathrm{spins} \mathrm{gives} \mathrm{a}$ common resonance with the itinerant electrons. ${ }^{78}$ This also means that the line width data in the $\mathrm{Na}$-doped sample are only reliable above $20 \mathrm{~K}$.

We identify the Pauli-like signal in the Na-doped FLG sample as coming from Na-doped graphene monolayers. It is based on the observation that $\mathrm{Na}$ cannot dope graphite; thus it is very probable that multilayer graphene is also inaccessible for it. This observation is in a full agreement with the Raman result, as it evidenced that Na doping of an FLG sample leaves a part of the sample undoped.

The ESR line width, $\Delta B$, data in Figure 4 can be used to directly obtain the spin-relaxation time through $1 / \tau_{\mathrm{s}}=1 / \gamma \Delta B$, where $\gamma=2 \pi \times 28.0 \mathrm{GHz} / \mathrm{T}$ is the electron gyromagnetic factor. The result is shown in Figure 5 along with reference

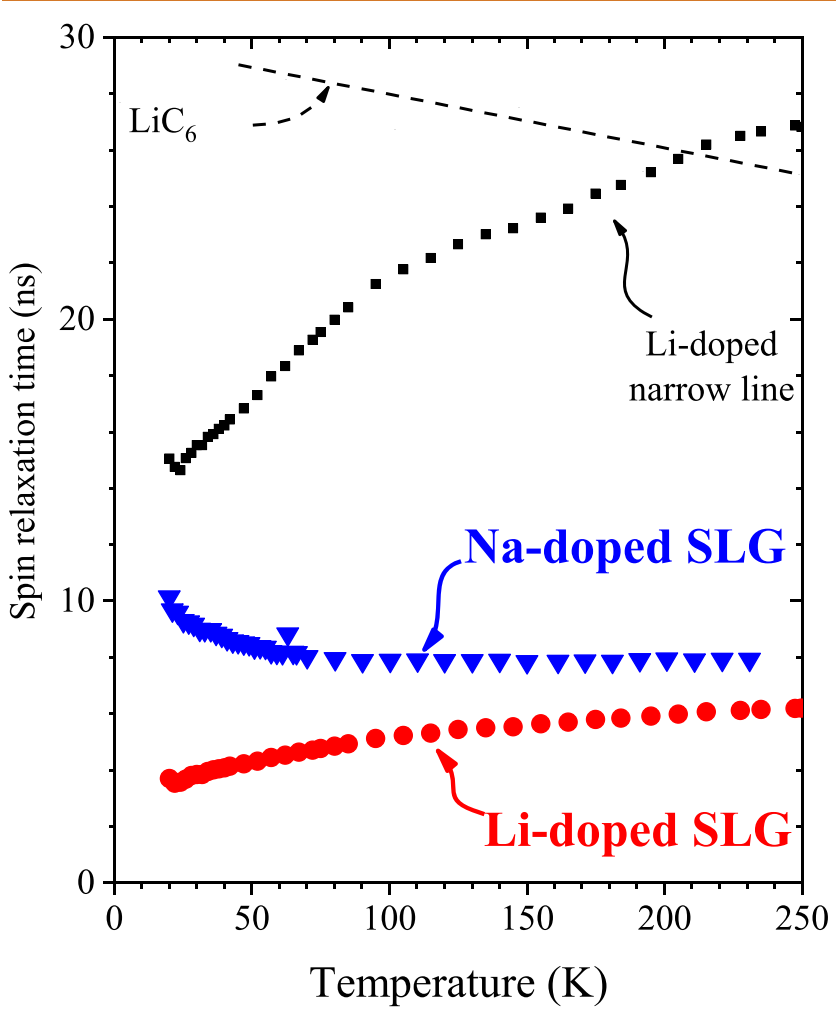

Figure 5. Spin-relaxation times of the Li- and Na-doped few-layer graphene above $20 \mathrm{~K}$. Dashed line shows data on the $\mathrm{LiC}_{6}$ intercalated graphite powder from ref 79.

data from $\mathrm{LiC}_{6}$-intercalated graphite powder from ref 79. The narrow component of the Li-doped FLG sample has a relaxation time that is similar to that in $\mathrm{LiC}_{6}$. It is therefore tempting to identify this component as coming from $\mathrm{Li}$ intercalated graphene multilayers and the broader component to Li-doped monolayers. The spin-relaxation time of this component is similar to that found for the $\mathrm{Na}$-doped graphene monolayers.

In principle, the temperature-dependent character of the spin-relaxation time also contains information about the relaxation mechanism, ${ }^{8,80}$ whether it can be described by the Elliott-Yafet ${ }^{20,21}$ or the D'yakonov-Perel theories. ${ }^{81}$ However, a proper theoretical description requires the knowledge of the temperature-dependent mobility and the local crystalline structure in these samples. Neither of these are available at present; therefore we cannot speculate on the underlying spinrelaxation mechanism.

Nevertheless, the striking observation is that the ESR line width at $230 \mathrm{~K}$, when translated to spin-relaxation times, gives $\sim 6.1 \mathrm{~ns}$ and $\sim 7.9 \mathrm{~ns}$ for lithium- and sodium-doped monolayer graphene, respectively. These values are even more surprising in view of the well-known heterogeneity of chemically exfoliated graphene and the synthesis method we employed. 
In fact, our figures are only surpassed by recent spin-transport experiments on carefully manufactured microscopic graphene samples, with ultrahigh mobility.

Early spin-transport experiments reported sub-nanosecond spin-relaxation times in graphene, ${ }^{11,14,15,82-85}$ which most probably originates from extrinsic effects. ${ }^{86}$ Advanced sample preparation and coating with hexagonal-BN resulted in improved values, ${ }^{87,88}$ with the longest spin-relaxation time values being $12.6 \mathrm{~ns}^{16}$ in single-layer graphene and $9.4 \mathrm{~ns}$ in bilayer graphene. $^{89}$

It is in fact astonishing that a chemically prepared sample, which is readily available in milligram quantities, displays the same magnitude for the spin-relaxation time as the above values, which are the result of advanced micromanipulation techniques, combined with van der Waals heterostructure engineering. The advanced techniques aim to decrease the defect concentration of individual graphene microstructures. However, bulk, top-down chemical methods may lead to higher quality flakes due to a finite probability that the flake defect concentration may even be smaller than those achieved by local techniques. As the sodium doping can only occur in high-quality single flakes, this may provoke a selectivity that further increases the measured spin-relaxation time, thus leading to the efficient manufacturing of future spintronics materials based on graphene.

\section{CONCLUSIONS}

In conclusion, we successfully synthesized lithium- and sodium-doped few-layer graphene in liquid ammonia solution. We found that $\mathrm{Na}$ dopes exclusively monolayer graphene that is present in the FLG sample. This is deduced from an overally weaker Na doping of the FLG samples, which is argued to be associated with the well-known inability of $\mathrm{Na}$ to intercalate graphite; thus multilayer graphene is also inaccessible for it. The obtained materials exhibit a clear change of color, a dominant Fano mode in the Raman spectrum, and ESR signals due to conduction electrons. These observations prove a successful intercalation and charge transfer from the alkali atoms toward graphene. Electron spin resonance spectroscopy indicates a spin-relaxation time of $6-8 \mathrm{~ns}$, which is comparable to the longest values found on ultrahigh-mobility graphene flakes using spin-transport methods. Given that we studied a relatively impure and bulk graphene material, these figures are encouraging for the spintronics applications of alkali atom doped graphene.

\section{METHODS}

Preparation of the Few Layer Graphene Starting Material. Few-layer graphene was prepared from saturation potassium-doped spherical graphite powder (SGN18, Future Carbon) using DMSO solvent for the wet chemical exfoliation as described previously. ${ }^{61,90,91}$ Chemical exfoliation was finalized using ultrasound tip sonication, which yields the best quality, as shown in a previous study. ${ }^{72}$ The properties of the starting material are well characterized by atomic force microscopy and Raman spectroscopy, which revealed that restacked few-layer graphene is also present in the sample. ${ }^{55}$ Prior to intercalation, the undoped FLG was heated to $400{ }^{\circ} \mathrm{C}$ for $30 \mathrm{~min}$ in high vacuum $\left(2 \times 10^{-6} \mathrm{mbar}\right)$ to remove any residual solvents. It was shown previously in refs 55 and 72 that this does not affect the morphology of the starting FLG.

Doping of Graphene with Lithium and Sodium. Lithium and sodium with a purity of $99.9+\%$ and $99.8 \%$ (from Sigma-Aldrich), respectively, were handled in an Ar glovebox $\left(\mathrm{O}_{2}, \mathrm{H}_{2} \mathrm{O}<0.1 \mathrm{ppm}\right)$. The lithium granules had a metallic color and were cut into smaller pieces to increase the surface. The sodium chunks were first thoroughly cleaned and then cut up. About $1 \mathrm{mg}$ of FLG and excess alkali metal of 1.1 or $2.2 \mathrm{mg}$ for lithium and sodium, respectively, were placed in a bulb-shaped quartz container (reaction chamber in the following) inside the glovebox. The bulb of the reaction chamber (hand-blown by a technician) had a diameter of $10 \mathrm{~mm}$, and it was connected to the vacuum line with a $5 \mathrm{~mm}$ diameter quartz tube. A $(\mathrm{Li}, \mathrm{Na}) \mathrm{C}_{6}$ stoichiometry (which is probably the upper limit of doping) would require $0.09 \mathrm{mg}$ and $0.24 \mathrm{mg}$ for the $\mathrm{Li}$ and $\mathrm{Na}$, respectively, for the $1 \mathrm{mg}$ of FLG. The unreacted mixture was then connected to a vacuum line with the help of a well-sealing valve and pumped to a vacuum better than $10^{-6}$ mbar.

The reaction chamber was placed in liquid nitrogen and gaseous ammonia, which was connected to the vacuum line and was rapidly (within a few seconds) condensed to the starting mixture of FLG and alkali metal. The rapid condensing is required to prevent the formation of side products. Following this, the reaction chamber is surrounded by an ethanol bath, which is kept at $-40{ }^{\circ} \mathrm{C}$ (below the $-33^{\circ} \mathrm{C}$ boiling point of ammonia at ambient pressure). Ethanol has a freezing point of $-114{ }^{\circ} \mathrm{C}$; thus it is well suited for this purpose. The reaction chamber, surrounded by the ethanol bath, was immersed in a bath sonicator, where it was intensively sonicated for $30 \mathrm{~min}$ to obtain a homogeneous doping of the few-layer graphene. The pressure of the chamber is monitored to avoid evaporation of the ammonia during the process. The dissolution of alkali metals in liquid ammonia can be followed by characteristic color changes: the solution changes its color to dark blue initially, immediately followed by a change to yellowbrownish. This marks the dissolution of the alkali metals: dark blue color indicates a low concentration of the dissolved alkali, and the yellow-brownish color is characteristic for a high concentration of dissolved alkali metals. ${ }^{92}$ Finally, the reaction chamber changes its color to a homogeneous brown, which indicates the doping of FLG with the alkali metals.

At the end of the intercalation, the solution is slowly heated to room temperature, when ammonia is evacuated. Subsequently, a heating of $200{ }^{\circ} \mathrm{C}$ is applied for $30 \mathrm{~min}$ to remove any absorbed ammonia and the unreacted alkali metal. The choice of $200{ }^{\circ} \mathrm{C}$ is delicate: on one hand it is above the melting temperature of the alkali metals $\left(181^{\circ} \mathrm{C}\right.$ for $\mathrm{Li}$ and $98^{\circ} \mathrm{C}$ for $\left.\mathrm{Na}\right)$, which allows for an efficient removal of the unreacted, excess alkali atoms, but on the other hand, it is below the onset of the formation temperature of alkali carbides (this occurs above $\sim 400{ }^{\circ} \mathrm{C}^{93}$ ). The reaction chambers were sealed off with a torch on the $5 \mathrm{~mm}$ quartz tube part.

Ammonia is known to react with alkali metal elements and forms amides. ${ }^{94,95}$ This side reaction is known to proceed fast when the ammonia is in the gas phase. However, this reaction channel is prohibited (or slowed down to the week time scale) when the ammonia is liquefied. Besides, the amides have well-known signatures in Raman spectroscopy ${ }^{96,97}$ in the form of a series of lines around $400-550 \mathrm{~cm}^{-1}$. These signals were used to monitor the content of this unwanted side product. We optimized the procedure to yield a material with no traces of amides. ${ }^{98}$ We emphasize at this point that while we refer to the material as "Na-doped FLG", Na dopes exclusively the monolayer graphene content inside the sample.

Raman Spectroscopy. Raman spectroscopy was performed on the as-prepared samples inside the same reaction chambers where the doping proceeds. A $514.5 \mathrm{~nm}$ wavelength laser excitation with 0.5 $\mathrm{mW}$ power was used to avoid laser-induced deintercalation ${ }^{56,99}$ or sample heating. Raman spectra were recorded on a modified broadband LabRAM spectrometer (Horiba Jobin-Yvon Inc.). The built-in interference filter was replaced by a beam splitter plate with $30 \%$ reflection and $70 \%$ transmission to allow for a broadband operation. ${ }^{100,101}$ Typically $0.5 \mathrm{~mW}$ laser powers were used with a built-in microscope (Olympus LMPlan $50 \times / 0.50$ inf./0/NN26.5), which yields an approximately $1 \mu \mathrm{m} \times 1 \mu \mathrm{m}$ spot size. Photographs were taken with an Olympus $10 \times / 50 \times$ objective, which is present on the LabRAM equipment.

Electron Spin Resonance. The reaction chambers were opened up inside an Ar glovebox and placed into quartz tubes with a $4 \mathrm{~mm}$ outer diameter. These tubes were then evacuated on the vacuum line 
to better than $2 \times 10^{-6}$ mbar, then filled with 20 mbar of He exchange gas for the cryogenic measurements and sealed permanently with a torch. Raman spectra of the materials were checked before and after this procedure to ensure that no changes occur during this operation. ESR measurements were performed on a Bruker Elexsys E500 X-band spectrometer equipped with an Oxford $\mathrm{He}$ flow cryostat. The temperature could be varied between 4 and $300 \mathrm{~K}$. Care was taken to avoid saturation and overmodulation of the observed signals, similarly to our previous works. ${ }^{54,102}$ The spectral parameters of each signal component are determined by fitting (derivative) Lorentzian and Dysonian curves, as is customary in the ESR literature. Due to the magnetic field modulation technique employed in ESR, the observed line shapes are the derivative of the following (with respect to the magnetic field, $B$ ):

$$
I(B)=A \frac{\Delta B \cos (\phi)+\left(B-B_{0}\right) \sin (\phi)}{\left(B-B_{0}\right)^{2}+\Delta B^{2}}
$$

where the ESR line shape, $I(B)$, is given as a function of the $B$ magnetic field, $A$ is proportional to the static spin susceptibility, $B_{0}$ is the line position, $\Delta B$ is the ESR line width, and $\phi$ is the mixing angle between the two types of curves (absorption and dispersion). The ESR line width is related to the $T_{2}$ spin-spin relaxation time as $\Delta B=$ $1 / \gamma T_{2}$ where $\gamma=2 \pi \times 28.0 \mathrm{GHz} / \mathrm{T}$ is the so-called electron gyromagnetic ratio.

Magnetometry with a Superconducting Quantum Interference Device. Samples inside the same He exchange gas filled quartz tubes were used for the SQUID measurement that were used in the ESR studies. We searched for traces of superconductivity in a standard MPMS SQUID magnetometer down to $2 \mathrm{~K}$ with a zero magnetic field cooling protocol, in small applied fields of 5-50 Oe, and also at $2 \mathrm{~K}$ in magnetic hysteresis loops.

\section{ASSOCIATED CONTENT}

\section{SI Supporting Information}

The Supporting Information is available free of charge at https://pubs.acs.org/doi/10.1021/acsnano.0c03191.

Details of the Raman spectra deconvolution, spectroscopic parameters of the Breit-Wigner-Fano line shapes, a discussion of the electron-phonon coupling, and the temperature-dependent $g$-factor as detected by electron spin resonance (PDF)

\section{AUTHOR INFORMATION}

\section{Corresponding Author}

F. Simon - Department of Physics, Budapest University of Technology and Economics and MTA-BME Lendület Spintronics Research Group (PROSPIN), H-1521 Budapest, Hungary; Laboratory of Physics of Complex Matter, Ecole Polytechnique Féderale de Lausanne, Lausanne CH-1015, Switzerland; 이이.org/0000-0001-9822-4309; Email: f.simon@eik.bme.hu

\section{Authors}

B. G. Márkus - Department of Physics, Budapest University of Technology and Economics and MTA-BME Lendület Spintronics Research Group (PROSPIN), H-1521 Budapest, Hungary; Laboratory of Physics of Complex Matter, Ecole Polytechnique Féderale de Lausanne, Lausanne CH-1015, Switzerland

P. Szirmai - Laboratory of Physics of Complex Matter, Ecole Polytechnique Féderale de Lausanne, Lausanne CH-1015, Switzerland; orcid.org/0000-0002-2841-5692

K. F. Edelthalhammer - Department of Chemistry and Pharmacy and Institute of Advanced Materials and Processes
(ZMP), University of Erlangen-Nürnberg, 91058 Erlangen, Germany

P. Eckerlein - Department of Chemistry and Pharmacy and Institute of Advanced Materials and Processes (ZMP), University of Erlangen-Nürnberg, 91058 Erlangen, Germany

A. Hirsch - Department of Chemistry and Pharmacy and Institute of Advanced Materials and Processes (ZMP), University of Erlangen-Nürnberg, 91058 Erlangen, Germany; (1) orcid.org/0000-0003-1458-8872

F. Hauke - Department of Chemistry and Pharmacy and Institute of Advanced Materials and Processes (ZMP), University of Erlangen-Nürnberg, 91058 Erlangen, Germany; (1) orcid.org/0000-0001-9637-7299

N. M. Nemes - GFMC, Unidad Asociada ICMM-CSIC "Laboratorio de Heteroestructuras con Aplicacion en Espintronica", Departamento de Fisica de Materiales Universidad Complutense de Madrid, 28040 Madrid, Spain

Julio C. Chacón-Torres - Yachay Tech University, School of Physical Sciences and Nanotechnology, 100119, Urcuquí, Ecuador and Universidad UTE, Facultad de Ciencias, Ingeniería y Construcción, 170147 Quito, Ecuador

B. Náfrádi - Laboratory of Physics of Complex Matter, Ecole Polytechnique Federale de Lausanne, Lausanne CH-1015, Switzerland; 이이이.org/0000-0001-9543-2970

L. Forró - Laboratory of Physics of Complex Matter, Ecole Polytechnique Féderale de Lausanne, Lausanne CH-1015, Switzerland

T. Pichler - Faculty of Physics, University of Vienna, Vienna A1090, Austria; (1) orcid.org/0000-0001-5377-9896

Complete contact information is available at: https://pubs.acs.org/10.1021/acsnano.0c03191

\section{Author Contributions}

B.G.M. performed the doping, Raman, and ESR studies under the supervision of F.S. P.Sz., B.N., and L.F. contributed to the Raman and ESR investigations. K.E. and P.E. prepared the starting FLG samples under the supervision of A.H. and F.H. SQUID measurements were performed by N.M.N. Raman studies and the analysis were performed by J.C.C-T. and T.P. All authors contributed to the writing of the manuscript.

\section{Notes}

The authors declare no competing financial interest.

\section{ACKNOWLEDGMENTS}

The authors are indebted to András Magyar for the preparation of the reaction chambers. We wish to kindly thank Prof. J. L. Martinez for performing the SQUID measurements. Support by the National Research, Development and Innovation Office of Hungary (NKFIH) Grant Nos. K119442 and 2017-1.2.1-NKP-2017-00001 are acknowledged. T.P. thanks the FWF (P27769-N20) for funding. K.F.E, P.E., F.H., and A.H. thank the German Science Foundation (DFG) for the financial support through Project-ID 182849149 - SFB 953. The work in Lausanne was supported by the Swiss National Science Foundation (Grant 200021 144419) and European Research Council (ERC) Advanced Grant Nr. 670918.

\section{REFERENCES}

(1) Korthauer, R. Lithium-Ion Batteries: Basics and Applications; Springer-Verlag GmbH: Berlin, Germany, 2018. 
(2) Emery, N.; Hérold, C.; d'Astuto, M.; Garcia, V.; Bellin, C.; Marêché, J. F.; Lagrange, P.; Loupias, G. Superconductivity of Bulk $\mathrm{CaC}_{6}$. Phys. Rev. Lett. 2005, 95, No. 087003.

(3) Emery, N.; Hérold, C.; Marêché, J.-F.; Lagrange, P. Synthesis and Superconducting Properties of $\mathrm{CaC}_{6}$. Sci. Technol. Adv. Mater. 2008, 9, No. 044102.

(4) Rosseinsky, M. J.; Ramirez, A. P.; Glarum, S. H.; Murphy, D. W.; Haddon, R. C.; Hebard, A. F.; Palstra, T. T. M.; Kortan, A. R.; Zahurak, S. M.; Makhija, A. V. Superconductivity at $28 \mathrm{~K}$ in $\mathrm{Rb}_{x} \mathrm{C}_{60}$. Phys. Rev. Lett. 1991, 66, 2830-2832.

(5) Jánossy, A.; Nemes, N.; Fehér, T.; Oszlányi, G.; Baumgartner, G.; Forró, L. Antiferromagnetic Resonance in the Linear Chain Conducting Polymers $\mathrm{RbC}_{60}$ and $\mathrm{CsC}_{60}$. Phys. Rev. Lett. 1997, 79, $2718-2721$.

(6) Ishii, H.; Kataura, H.; Shiozawa, H.; Yoshioka, H.; Otsubo, H.; Takayama, Y.; Miyahara, T.; Suzuki, S.; Achiba, Y.; Nakatake, M.; Narimura, T.; Higashiguchi, M.; Shimada, K.; Taniguchi, M. Direct Observation of Tomonaga-Luttinger-Liquid State in Carbon Nanotubes at Low Temperatures. Nature 2003, 426, 540-544.

(7) Rauf, H.; Pichler, T.; Knupfer, M.; Fink, J.; Kataura, H. Transition from a Tomonaga-Luttinger Liquid to a Fermi Liquid in Potassium-Intercalated Bundles of Single-Wall Carbon Nanotubes. Phys. Rev. Lett. 2004, 93, No. 096805.

(8) Žutić, I.; Fabian, J.; Das Sarma, S. Spintronics: Fundamentals and Applications. Rev. Mod. Phys. 2004, 76, 323-410.

(9) Wolf, S. A.; Awschalom, D. D.; Buhrman, R. A.; Daughton, J. M.; von Molnár, S.; Roukes, M. L.; Chtchelkanova, A. Y.; Treger, D. M. Spintronics: A Spin-Based Electronics Vision for the Future. Science 2001, 294, 1488-1495.

(10) Wu, M. W.; Jiang, J. H.; Weng, M. Q. Spin Dynamics in Semiconductors. Phys. Rep. 2010, 493, 61-236.

(11) Tombros, N.; Józsa, C.; Popinciuc, M.; Jonkman, H. T.; van Wees, B. J. Electronic Spin Transport and Spin Precession in Single Graphene Layers at Room Temperature. Nature 2007, 448, 571-574.

(12) Han, W.; Kawakami, R. K. Spin Relaxation in Single-Layer and Bilayer Graphene. Phys. Rev. Lett. 2011, 107, No. 047207.

(13) Yang, T.-Y.; Balakrishnan, J.; Volmer, F.; Avsar, A.; Jaiswal, M.; Samm, J.; Ali, S. R.; Pachoud, A.; Zeng, M.; Popinciuc, M.; Güntherodt, G.; Beschoten, B.; Özyilmaz, B. Observation of Long Spin-Relaxation Times in Bilayer Graphene at Room Temperature. Phys. Rev. Lett. 2011, 107, No. 047206.

(14) Avsar, A.; Yang, T.-Y.; Bae, S.; Balakrishnan, J.; Volmer, F.; Jaiswal, M.; Yi, Z.; Ali, S. R.; Güntherodt, G.; Hong, B. H.; Beschoten, B.; Özyilmaz, B. Toward Wafer Scale Fabrication of Graphene Based Spin Valve Devices. Nano Lett. 2011, 11, 2363-2368.

(15) Han, W.; Chen, J.-R.; Wang, D.; McCreary, K. M.; Wen, H.; Swartz, A. G.; Shi, J.; Kawakami, R. K. Spin Relaxation in Single-Layer Graphene with Tunable Mobility. Nano Lett. 2012, 12, 3443-3447.

(16) Drögeler, M.; Franzen, C.; Volmer, F.; Pohlmann, T.; Banszerus, L.; Wolter, M.; Watanabe, K.; Taniguchi, T.; Stampfer, C.; Beschoten, B. Spin Lifetimes Exceeding 12 ns in Graphene Nonlocal Spin Valve Devices. Nano Lett. 2016, 16, 3533-3539.

(17) Kochan, D.; Gmitra, M.; Fabian, J. Spin Relaxation Mechanism in Graphene: Resonant Scattering by Magnetic Impurities. Phys. Rev. Lett. 2014, 112, 116602.

(18) Feher, G.; Kip, A. F. Electron Spin Resonance Absorption in Metals. I. Experimental. Phys. Rev. 1955, 95, 337-348.

(19) Dyson, F. J. Electron Spin Resonance Absorption in Metals. II. Theory of Electron Diffusion and the Skin Effect. Phys. Rev. 1955, 98, 349-359.

(20) Elliott, R. J. Theory of the Effect of Spin-Orbit Coupling on Magnetic Resonance in Some Semiconductors. Phys. Rev. 1954, 96, 266-279.

(21) Yafet, Y. $g$-Factors and Spin-Lattice Relaxation of Conduction Electrons. Solid State Phys. 1963, 14, 1-98.

(22) Dresselhaus, M. S.; Dresselhaus, G. Intercalation Compounds of Graphite. Adv. Phys. 1981, 30, 139-326.

(23) Jung, N.; Kim, B.; Crowther, A. C.; Kim, N.; Nuckolls, C.; Brus, L. Optical Reflectivity and Raman Scattering in Few-Layer-Thick
Graphene Highly Doped by K and Rb. ACS Nano 2011, 5, 57085716.

(24) Howard, C. A.; Dean, M. P. M.; Withers, F. Phonons in Potassium-Doped Graphene: The Effects of Electron-Phonon Interactions, Dimensionality, and Adatom Ordering. Phys. Rev. B: Condens. Matter Mater. Phys. 2011, 84, 241404R.

(25) Kumar, A.; Reddy, A. L. M.; Mukherjee, A.; Dubey, M.; Zhan, X.; Singh, N.; Ci, L.; Billups, W. E.; Nagurny, J.; Mital, G.; Ajayan, P. M. Direct Synthesis of Lithium-Intercalated Graphene for Electrochemical Energy Storage Application. ACS Nano 2011, 5, 4345-4349.

(26) Xue, M.; Chen, G.; Yang, H.; Zhu, Y.; Wang, D.; He, J.; Cao, T. Superconductivity in Potassium-Doped Few-Layer Graphene. J. Am. Chem. Soc. 2012, 134, 6536-6539.

(27) Parret, R.; Paillet, M.; Huntzinger, J.-R.; Nakabayashi, D.; Michel, T.; Tiberj, A.; Sauvajol, J.-L.; Zahab, A. A. In Situ Raman Probing of Graphene over a Broad Doping Range Upon Rubidium Vapor Exposure. ACS Nano 2013, 7, 165-173.

(28) Zhou, E.; Xi, J.; Liu, Y.; Xu, Z.; Guo, Y.; Peng, L.; Gao, W.; Ying, J.; Chen, Z.; Gao, C. Large-Area Potassium-Doped Highly Conductive Graphene Films for Electromagnetic Interference Shielding. Nanoscale 2017, 9, 18613-18618.

(29) Pervan, P.; Lazić, P. Adsorbed or Intercalated: $\mathrm{Na}$ on Graphene/Ir(111). Phys. Rev. Mater. 2017, 1, No. 044202.

(30) Wang, G.; Shen, X.; Yao, J.; Park, J. Graphene Nanosheets for Enhanced Lithium Storage in Lithium Ion Batteries. Carbon 2013, 47, 2049-2053.

(31) Yang, X.; Cheng, C.; Wang, Y.; Qiu, L.; Li, D. Liquid-Mediated Dense Integration of Graphene Materials for Compact Capacitive Energy Storage. Science 2013, 341, 534-537.

(32) Mahmood, N.; Zhang, C.; Yin, H.; Hou, Y. Graphene-Based Nanocomposites for Energy Storage and Conversion in Lithium Batteries, Supercapacitors and Fuel Cells. J. Mater. Chem. A 2014, 2, $15-32$.

(33) Medarde, M.; Mena, M.; Gavilano, J. L.; Pomjakushina, E.; Sugiyama, J.; Kamazawa, K.; Pomjakushin, V. Y.; Sheptyakov, D.; Batlogg, B.; Ott, H. R.; Månsson, M.; Juranyi, F. 1D to $2 \mathrm{D} \mathrm{Na}^{+}$Ion Diffusion Inherently Linked to Structural Transitions in $\mathrm{Na}_{0.7} \mathrm{CoO}_{2}$. Phys. Rev. Lett. 2013, 110, 266401.

(34) Barpanda, P.; Oyama, G.; ichi Nishimura, S.; Chung, S.-C.; Yamada, A. A 3.8-V Earth-Abundant Sodium Battery Electrode. Nat. Commun. 2014, 5, 4358.

(35) Ares, P.; Palacios, J. J.; Abellán, G.; Gómez-Herrero, J.; Zamora, F. Recent Progress on Antimonene: A New Bidimensional Material. Adv. Mater. 2018, 30, 1703771.

(36) Tian, W.; Zhang, S.; Huo, C.; Zhu, D.; Li, Q.; Wang, L.; Ren, X.; Xie, L.; Guo, S.; Chu, P. K.; Zeng, H.; Huo, K. Few-Layer Antimonene: Anisotropic Expansion and Reversible Crystalline-Phase Evolution Enable Large-Capacity and Long-Life Na-Ion Batteries. ACS Nano 2018, 12, 1887-1893.

(37) Asher, R. C.; Wilson, S. A. Lamellar Compound of Sodium with Graphite. Nature 1958, 181, 409-410.

(38) Asher, R. C. A Lamellar Compound of Sodium and Graphite. J. Inorg. Nucl. Chem. 1959, 10, 238-249.

(39) Metrot, A.; Guerard, D.; Billaud, D.; Herold, A. New Results about the Sodium-Graphite System. Synth. Met. 1980, 1, 363-369.

(40) Akuzawa, N.; Yoshioka, J.; Ozaki, C.; Tokuda, M.; Ohkura, K.; Soneda, Y. Preparation and Characterization of Sodium-Graphite Intercalation Compounds. Mol. Cryst. Liq. Cryst. 2002, 388, 1-7.

(41) Lenchuk, O.; Adelhelm, P.; Mollenhauer, D. New Insights into the Origin of Unstable Sodium Graphite Intercalation Compounds. Phys. Chem. Chem. Phys. 2019, 21, 19378-19390.

(42) Choucair, M.; Thordarson, P.; Stride, J. A. Gram-Scale Production of Graphene Based on Solvothermal Synthesis and Sonication. Nat. Nanotechnol. 2009, 4, 30.

(43) Pramudita, J.; Rawal, A.; Choucair, M.; Pontiroli, D.; Magnani, G.; Gaboardi, M.; Ricco, M.; Sharma, N. Mechanisms of Sodium Insertion/Extraction on the Surface of Defective Graphenes ACS Appl. Mater. Interfaces 2017, 9, 431. 
(44) Quan, B.; Jin, A.; Yu, S.-H.; Kang, S. M.; Jeong, J.; Abruña, H. D.; Jin, L.; Piao, Y.; Sung, Y.-E. Solvothermal-Derived S-Doped Graphene as an Anode Material for Sodium-Ion Batteries. Adv. Sci. 2018, 5, 1700880.

(45) Beguin, F.; Setton, R.; Beguin, F.; Setton, R.; Hamwi, A.; Touzain, P. The Reversible Intercalation of Tetrahydrofuran in Some Graphite-Alkali Metal Lamellar Compounds. Mater. Sci. Eng. 1979, 40, 167-173.

(46) Mizutani, Y.; Ihara, E.; Abe, T.; Asano, M.; Harada, T.; Ogumi, Z.; Inaba, M. Preparation of Alkali Metal Graphite Intercalation Compounds in Organic Solvents. J. Phys. Chem. Solids 1996, 57, 799803.

(47) Mizutani, Y.; Abe, T.; Inaba, M.; Ogumi, Z. Creation of Nanospaces by Intercalation of Alkali Metals into Graphite in Organic Solutions. Synth. Met. 2001, 125, 153-159.

(48) Murphy, D. W.; Rosseinsky, M. J.; Fleming, R. M.; Tycko, R.; Ramirez, A. P.; Haddon, R. C.; Siegrist, T.; Dabbagh, G.; Tully, J. C.; Walstedt, R. E. Synthesis and Characterization of Alkali Metal Fullerides: $\mathrm{A}_{\mathrm{x}} \mathrm{C}_{60}$. J. Phys. Chem. Solids 1992, 53, 1321-1332.

(49) Buffinger, D. R.; Ziebarth, R. P.; Stenger, V. A.; Recchia, C.; Pennington, C. H. Rapid and Efficient Synthesis of Alkali Metal- $\mathrm{C}_{60}$ Compounds in Liquid Ammonia. J. Am. Chem. Soc. 1993, 115, 92679270

(50) Dahlke, P.; Denning, M. S.; Henry, P. F.; Rosseinsky, M. J. Superconductivity in Expanded FCC C 60 Fullerides. J. Am. Chem. Soc. 2000, 122, 12352-12361.

(51) Kumar, A.; Reddy, A. L. M.; Mukherjee, A.; Dubey, M.; Zhan, X.; Singh, N.; Ci, L.; Billups, W. E.; Nagurny, J.; Mital, G.; Ajayan, P. M. Direct Synthesis of Lithium-Intercalated Graphene for Electrochemical Energy Storage Application. ACS Nano 2011, 5, 4345-4349.

(52) Ganin, A. Y.; Takabayashi, Y.; Jeglič, P.; Arčon, D.; Potočnik, A.; Baker, P. J.; Ohishi, Y.; McDonald, M. T.; Tzirakis, M. D.; McLennan, A.; Darling, G. R.; Takata, M.; Rosseinsky, M. J.; Prassides, K. Polymorphism Control of Superconductivity and Magnetism in $\mathrm{Cs}_{3} \mathrm{C}_{60}$ Close to the Mott Transition. Nature 2010, $466,221-225$.

(53) Hirsch, A. The Chemistry of the Fullerenes; Wiley-VCH Verlag GmbH \& Co. KGaA: Weinheim, Germany, 2002.

(54) Szirmai, P.; Márkus, B. G.; Dóra, B.; Fábián, G.; Koltai, J.; Zólyomi, V.; Kürti, J.; Náfrádi, B.; Forró, L.; Pichler, T.; Simon, F. Doped Carbon Nanotubes as a Model System of Biased Graphene. Phys. Rev. B: Condens. Matter Mater. Phys. 2017, 96, No. 075133.

(55) Szirmai, P.; Márkus, B. G.; Chacón-Torres, J. C.; Eckerlein, P.; Edelthalhammer, K.; Englert, J. M.; Mundloch, U.; Hirsch, A.; Hauke, F.; Náfrádi, B.; Forró, L.; Kramberger, C.; Pichler, T.; Simon, F. Characterizing the Maximum Number of Layers in Chemically Exfoliated Graphene. Sci. Rep. 2019, 9, 19480.

(56) Chacón-Torres, J. C.; Ganin, A. Y.; Rosseinsky, M. J.; Pichler, T. Raman Response of Stage-1 Graphite Intercalation Compounds Revisited. Phys. Rev. B: Condens. Matter Mater. Phys. 2012, 86, No. 075406.

(57) Udod, I. A.; Genchel, V. K. Synthesis Under High Pressure and X-Rays Study Graphite Intercalation Compounds of Sodium. High Pressure Res. 1992, 8, 581-586.

(58) Oszlányi, G.; Baumgartner, G.; Faigel, G.; Forró, L. $\mathrm{Na}_{4} \mathrm{C}_{60}$ : An Alkali Intercalated Two-Dimensional Polymer. Phys. Rev. Lett. 1997, $78,4438-4441$

(59) Brouet, V.; Alloul, H.; Le, T.-N.; Garaj, S.; Forró, L. Role of Dynamic Jahn-Teller Distortions in $\mathrm{Na}_{2} \mathrm{C}_{60}$ and $\mathrm{Na}_{2} \mathrm{CsC}_{60}$ Studied by NMR. Phys. Rev. Lett. 2001, 86, 4680-4683.

(60) Brouet, V.; Alloul, H.; Garaj, S.; Forró, L. Gaps and Excitations in Fullerides with Partially Filled Bands: NMR Study of $\mathrm{Na}_{2} \mathrm{C}_{60}$ and $\mathrm{K}_{4} \mathrm{C}_{60}$. Phys. Rev. B: Condens. Matter Mater. Phys. 2002, 66, 155122.

(61) Englert, J. M.; Dotzer, C.; Yang, G.; Schmid, M.; Papp, C.; Gottfried, J. M.; Steinrück, H.-P.; Spiecker, E.; Hauke, F.; Hirsch, A. Covalent Bulk Functionalization of Graphene. Nat. Chem. 2011, 3, 279-286.

(62) Fano, U. Effects of Configuration Interaction on Intensities and Phase Shifts. Phys. Rev. 1961, 124, 1866-1878.
(63) Kuzmany, H. Solid-State Spectroscopy, An Introduction, 2nd ed.; Springer-Verlag: Vienna, Austria, 2009.

(64) Chacón-Torres, J. C.; Wirtz, L.; Pichler, T. Manifestation of Charged and Strained Graphene Layers in the Raman Response of Graphite Intercalation Compounds. ACS Nano 2013, 7, 9249-9259.

(65) Kuzmany, H.; Matus, M.; Burger, B.; Winter, J. Raman Scattering in $\mathrm{C}_{60}$ Fullerenes and Fullerides. Adv. Mater. 1994, 6, 731745.

(66) Rao, A. M.; Eklund, P. C.; Bandow, S.; Thess, A.; Smalley, R. E. Evidence for Charge Transfer in Doped Carbon Nanotube Bundles from Raman Scattering. Nature 1997, 388, 257-259.

(67) Eklund, P. C.; Dresselhaus, G.; Dresselhaus, M. S.; Fischer, J. E. Raman Scattering from Inplane Lattice Modes in Low-Stage GraphiteAlkali-Metal Compounds. Phys. Rev. B 1977, 16, 3330-3333.

(68) Weller, T. E.; Ellerby, M.; Saxena, S. S.; Smith, R. P.; Skipper, N. T. Superconductivity in the Intercalated Graphite Compounds $\mathrm{C}_{6} \mathrm{Yb}$ and $\mathrm{C}_{6} \mathrm{Ca}$. Nat. Phys. 2005, 1, 39-41.

(69) Slichter, C. P. Principles of Magnetic Resonance, 2nd ed.; Springer: New York, USA, 1989.

(70) Nemes, N. M.; Fischer, J. E.; Baumgartner, G.; Forró, L.; Fehér, T.; Oszlányi, G.; Simon, F.; Jánossy, A. Conduction-Electron Spin Resonance in the Superconductor $\mathrm{K}_{3} \mathrm{C}_{60}$. Phys. Rev. B: Condens. Matter Mater. Phys. 2000, 61, 7118-7121.

(71) Galambos, M.; Fábián, G.; Simon, F.; Ćirić, L.; Forró, L.; Korecz, L.; Rockenbauer, A.; Koltai, J.; Zólyomi, V.; Rusznyák, Á.; Kürti, J.; Nemes, N. M.; Dóra, B.; Peterlik, H.; Pfeiffer, R.; Kuzmany, H.; Pichler, T. Identifying the Electron Spin Resonance of Conduction Electrons in Alkali Doped SWCNTs. Phys. Status Solidi B 2009, 246, 2760-2763.

(72) Márkus, B. G.; Simon, F.; Chacón-Torres, J. C.; Reich, S.; Szirmai, P.; Náfrádi, B.; Forró, L.; Pichler, T.; Vecera, P.; Hauke, F.; Hirsch, A. Transport, Magnetic and Vibrational Properties of Chemically Exfoliated Few-Layer Graphene. Phys. Status Solidi B 2015, 252, 2438-2443.

(73) Szirmai, P.; Fábián, G.; Koltai, J.; Náfrádi, B.; Forró, L.; Pichler, T.; Williams, O. A.; Mandal, S.; Bäuerle, C.; Simon, F. Observation of Conduction Electron Spin Resonance in Boron-Doped Diamond. Phys. Rev. B: Condens. Matter Mater. Phys. 2013, 87, 195132.

(74) Jánossy, A.; Chauvet, O.; Pekker, S.; Cooper, J. R.; Forró, L. Conduction Electron Spin Resonance in $\mathrm{Rb}_{3} \mathrm{C}_{60}$. Phys. Rev. Lett. 1993, 71, 1091-1094.

(75) Claye, A. S.; Nemes, N. M.; Jánossy, A.; Fischer, J. E. Structure and Electronic Properties of Potassium-Doped Single-Wall Carbon Nanotubes. Phys. Rev. B: Condens. Matter Mater. Phys. 2000, 62, R4845-R4848.

(76) Walmsley, L. Translating Conduction-Electron Spin-Resonance Lines into Lorentzian Lines. J. Magn. Reson., Ser. A 1996, 122, 209213.

(77) Djokić, D. M.; Stepanenko, D.; Dohčević-Mitrović, Z. Extreme Conduction Electron Spin Resonance: $A / B \rightarrow 53 \sqrt{3} / 4$, the Universal Limit of Lineshape Asymmetry Ratio. J. Magn. Magn. Mater. 2019, $491,165616$.

(78) Barnes, S. E. Theory of Electron Spin Resonance of Magnetic Ions in Metals. Adv. Phys. 1981, 30, 801-938.

(79) Lauginie, P.; Estrade, H.; Conard, J.; Guérard, D.; Lagrange, P.; El Makrini, M. Graphite Lamellar Compounds ESR Studies. Physica B $+C$ 1980, 99, 514-520.

(80) Tsymbal, E. Y.; Žćc, I. Handbook of Spin Transport and Magnetism; Taylor \& Francis: New York, USA, 2012.

(81) Dyakonov, M. I.; Perel, V. I. Spin Relaxation of Conduction Electrons in Noncentrosymmetric Semiconductors. Sov. Phys. Solid State Phys. 1971, 13, 3023.

(82) Han, W.; Pi, K.; McCreary, K. M.; Li, Y.; Wong, J. J. I.; Swartz, A. G.; Kawakami, R. K. Tunneling Spin Injection into Single Layer Graphene. Phys. Rev. Lett. 2010, 105, 167202.

(83) Yang, T.-Y.; Balakrishnan, J.; Volmer, F.; Avsar, A.; Jaiswal, M.; Samm, J.; Ali, S. R.; Pachoud, A.; Zeng, M.; Popinciuc, M.; Güntherodt, G.; Beschoten, B.; Özyilmaz, B. Observation of Long 
Spin-Relaxation Times in Bilayer Graphene at Room Temperature. Phys. Rev. Lett. 2011, 107, No. 047206.

(84) Han, W.; Kawakami, R. K. Spin Relaxation in Single-Layer and Bilayer Graphene. Phys. Rev. Lett. 2011, 107, No. 047207.

(85) Volmer, F.; Drögeler, M.; Maynicke, E.; von den Driesch, N.; Boschen, M. L.; Güntherodt, G.; Beschoten, B. Role of $\mathrm{MgO}$ Barriers for Spin and Charge Transport in $\mathrm{Co} / \mathrm{MgO} / \mathrm{Graphene}$ Nonlocal Spin-Valve Devices. Phys. Rev. B: Condens. Matter Mater. Phys. 2013, $88,161405$.

(86) Kochan, D.; Gmitra, M.; Fabian, J. Spin Relaxation Mechanism in Graphene: Resonant Scattering by Magnetic Impurities. Phys. Rev. Lett. 2014, 112, 116602.

(87) Drögeler, M.; Volmer, F.; Wolter, M.; Terrés, B.; Watanabe, K.; Taniguchi, T.; Güntherodt, G.; Stampfer, C.; Beschoten, B. Nanosecond Spin Lifetimes in Single- and Few-Layer GraphenehBN Heterostructures at Room Temperature. Nano Lett. 2014, 14, $6050-6055$.

(88) Guimarães, M. H. D.; Zomer, P. J.; Ingla-Aynés, J.; Brant, J. C.; Tombros, N.; van Wees, B. J. Controlling Spin Relaxation in Hexagonal BN-Encapsulated Graphene with a Transverse Electric Field. Phys. Rev. Lett. 2014, 113, No. 086602.

(89) Leutenantsmeyer, J. C.; Ingla-Aynés, J.; Fabian, J.; van Wees, B. J. Observation of Spin-Valley-Coupling-Induced Large Spin-Lifetime Anisotropy in Bilayer Graphene. Phys. Rev. Lett. 2018, 121, 127702.

(90) Vecera, P.; Edelthalhammer, K.; Hauke, F.; Hirsch, A. Reductive Arylation of Graphene: Insights into a Reversible Carbon Allotrope Functionalization Reaction. Phys. Status Solidi B 2014, 251, $2536-2540$

(91) Vecera, P.; Chacón-Torres, J. C.; Pichler, T.; Reich, S.; Soni, H. R.; Görling, A.; Edelthalhammer, K.; Peterlik, H.; Hauke, F.; Hirsch, A. Precise Determination of Graphene Functionalization by In Situ Raman Spectroscopy. Nat. Commun. 2017, 8, 15192.

(92) Greenwood, N. N.; Earnshaw, A. Chemistry of the Elements; Butterworth: London, UK, 1998.

(93) Basu, S.; Zeller, C.; Flanders, P. J.; Fuerst, C. D.; Johnson, W. D.; Fischer, J. E. Synthesis and Properties of Lithium-Graphite Intercalation Compounds. Mater. Sci. Eng. 1979, 38, 275-283.

(94) Bergstrom, F. W.; Fernelius, W. C. The Chemistry of the Alkali Amides. Chem. Rev. 1933, 12, 43-179.

(95) Juza, R. Amide der Alkali- und Erdalkalimetalle. Angew. Chem. 1964, 76, 290-300.

(96) Cunningham, P. T.; Maroni, V. A. Laser Raman Spectra of Solid and Molten $\mathrm{NaNH}_{2}$ : Evidence for Hindered Rotation of the $\mathrm{NH}_{2}^{-1}$ Ion. J. Chem. Phys. 1972, 57, 1415-1418.

(97) Liu, A.; Song, Y. In Situ High-Pressure Study of Sodium Amide by Raman and Infrared Spectroscopies. J. Phys. Chem. B 2011, 115, $7-13$.

(98) Márkus, B. G.; Szirmai, P.; Kollarics, S.; Náfrádi, B.; Forró, L.; Chacón-Torres, J. C.; Pichler, T.; Simon, F. Improved Alkali Intercalation of Carbonaceous Materials in Ammonia Solution. Phys. Status Solidi B 2019, 256, 1900324.

(99) Nemanich, R. J.; Solin, S. A.; Gerard, D. Raman Scattering from Intercalated Donor Compounds of Graphite. Phys. Rev. B 1977, 16, 2965-2972.

(100) Fábián, G.; Kramberger, C.; Friedrich, A.; Simon, F.; Pichler, T. A Broadband and High Throughput Single-Monochromator Raman Spectrometer: Application for Single-Wall Carbon Nanotubes. Rev. Sci. Instrum. 2011, 82, 039901.

(101) Fábián, G.; Kramberger, C.; Friedrich, A.; Simon, F.; Pichler, T. Adaptation of a Commercial Raman Spectrometer for Multiline and Broadband Laser Operation. Phys. Status Solidi B 2011, 248, 2581-2584.

(102) Szirmai, P.; Fábián, G.; Dóra, B.; Koltai, J.; Zólyomi, V.; Kürti, J.; Nemes, N. M.; Forró, L.; Simon, F. Density of States Deduced from ESR Measurements on Low-Dimensional Nanostructures; Benchmarks to Identify the ESR Signals of Graphene and SWCNTs. Phys. Status Solidi B 2011, 248, 2688-2691. 